\title{
An Airline Scheduling Model and Solution Algorithms
}

\author{
Ahmed Thanyan AL-Sultan ${ }^{1, a}$, Fumio Ishioka ${ }^{b}$, Koji Kurihara ${ }^{a}$ \\ ${ }^{a}$ Graduate School of Environmental Science, Okayama University \\ ${ }^{b}$ School of Law, Okayama University
}

\begin{abstract}
The rapid development of airlines, has made airports busier and more complicated. The assignment of scheduled to available gates is a major issue for daily airline operations. We consider the over-constrained airport gate assignment problem(AGAP) where the number of flights exceeds the number of available gates, and where the objectives are to minimize the number of ungated flights and the total walking distance or connection times. The procedures used in this project are to create a mathematical model formulation to identify decision variablesto identify, constraints and objective functions. In addition, we will consider in the AGAP the size of each gate in the terminal and also the towing process for the aircraft. We will use a greedy algorithm to solve the problem. The greedy algorithm minimizes ungated flights while providing initial feasible solutions that allow flexibility in seeking good solutions, especially in case when flight schedules are dense in time. Experiments conducts give good results.
\end{abstract}

Keywords: Operations research, mathematical programming, greedy algorithm, 0-1 integer programming, Monte Carlo simulation.

\section{Introduction}

The problem of assigning gates to flight arrivals and archers is an important decision problem in daily operations at major airports all over the world. Strong competition between airlines and the increasing demands of passengers for increased comfort has made the measures of quality in their decisions at an airport as important performance indices of airport management. This is why the mathematical modeling of this problem and the application of Operations Research(OR) methods to solve those models have been studied widely in OR literature. The common characteristics of busy international airports usually involve serving a large number of different airlines, a large number of flights over day, and accommodating various types of planes.

Much work has centered on the gate assigning problem with the objective of minimizing distance cost (or variants of this). One of the first attempts to use quantitative means to minimize intra-terminal travel into a design process was given by Braaksma and Shortreed (1971). The assignment of aircraft to gates that minimize travel distances, is an easily motivated and understood problem but a difficult one to solve. The total passenger walking distance is based on passenger embarkation and disembarkation volumes, transfer passenger volumes, gate to gate distances, check in to gate distances and aircraft to gate assignments. In the gate assignment problem, the cost associated with the placing of an aircraft at a gate depends on the distances from key facilities as well as the relations between these facilities. The basic gate assignment problem is quadratic assignment problem as shown to be NP-hard in Obata (1979). Babic et al. (1984) formulated the gate assignment problem as linear 0-1

\footnotetext{
${ }^{1}$ Corresponding author: Professor, Graduate School of Environmental Science, Okayama University, 3-1-1 Tsushimanaka Okayama 700-8530, Japan. E-mail: alsultan@ems.okayama-u.ac.jp
} 
IP. A branch and bound algorithm is used to find the optimal solution where transfer passengers are not considered. Haghani and Chen (1998) proposed an integer programming formulation of the gate assignment problem and heuristic solution procedure for solving the problem. The multiple objective model for the gate assignments were proposed in Yan and Huo (2001). Where the model is formulated as a multiple objective 0-1 integer program. Network model (Yan and Chang, 1998) and simulation models (Cheng, 1998a, b) were also proposed to formulate the problem. Since the gate assignment problem is NP-hard, various heuristic approaches have been suggested by search, e.g. Haghnani and Chen (1998). Proposed a heuristic that assigns successive flights parking at the same gate when there is no overlapping, flights are assigned based on the shortest walking distance coefficients. $\mathrm{Xu}$ and Bailey (2001) provide a Tabu search meta-heuristic to solve the problem. The algorithm exploits the special properties of different types of neighborhood moves, and creates highly effective candidate list strategies. The work of Yan et al. (2008) considered stochastic disturbances in the daily passenger demand that occur in actual operations. They established a stochastic-demand flight scheduling model, SDFSM. Two heuristic algorithms, based on arc-based and route based strategies, were developed to solve the SDFSM. In addition, previous work (Ding et el., 2004) has considered the over constrained gate assignment problem which addressed both the objectives of minimizing the number of ungated aircraft while minimizing low total walking distance. In the work of AL-Sultan et el. (2010), some of the assumptions has been added and changed for a previous work of Ding et el. (2004) such as considering in the airport gate assignment problem the size of each gate in the terminal and also the towing process for the aircraft; however, the data collection was for one day only. In the current project, the data collection is for one week. In addition, analysis will be added for the buffer time that is the time that locks the aircraft gate after departure. To the best of our knowledge, no previous work has considered the over constrained gate assignment problem. In particular, no previous work has addressed both the objectives of minimizing the number of ungated aircraft while minimizing the total walking distance. In addition, we inserted the terminal gate sizes, the towing process, and aircraft capacity.

The Airport gate assignment problem(AGAP) seeks to a find feasible flight to gate assignments so that the number of the flights that need be assigned to the apron and total passenger connection times, as can be proxies for walking distances, are minimized. In this paper, we discuss a greedy algorithm that minimizes the number of flights not assigned to gates.

We will apply our model to Kuwait International Airport(KIA) that has become busier after applying for the "Open Skies" policy that applies to passenger and cargo operations, forms an essential part of the Kuwait government's latest initiative to promote the state as a major center for financial, commercial and economic activities in the Gulf Region. KIA already serves more than 50 airlines currently connected to Kuwait with over 100 international destinations; in addition, there exists considerable room for expansion. In this project, we will use actual aircraft's arrival and departure schedules. The number of passengers for each aircraft will be generated randomly using the Monte Carlo method.

\section{The Nature of KIA's Gate Terminals}

Kuwait international airport has one terminal that has ten gates for the aircraft. The gate numbers are $1,2,3,4,5,21,22,24,25$ and 26. Figure 1 describes the shape of the terminal. Next to the terminal there are stands (aprons) for the ungated flights, there are several locations for aircraft stands such as the cargo flight stand area, the VIP or privet flight stand area, and the regular stand area which is used mostly for the ungated commercial aircraft.

Table 1 presents the actual schedule (daily movement) for a sample of the arrival and departure flights for a specific day. The schedule contents: 


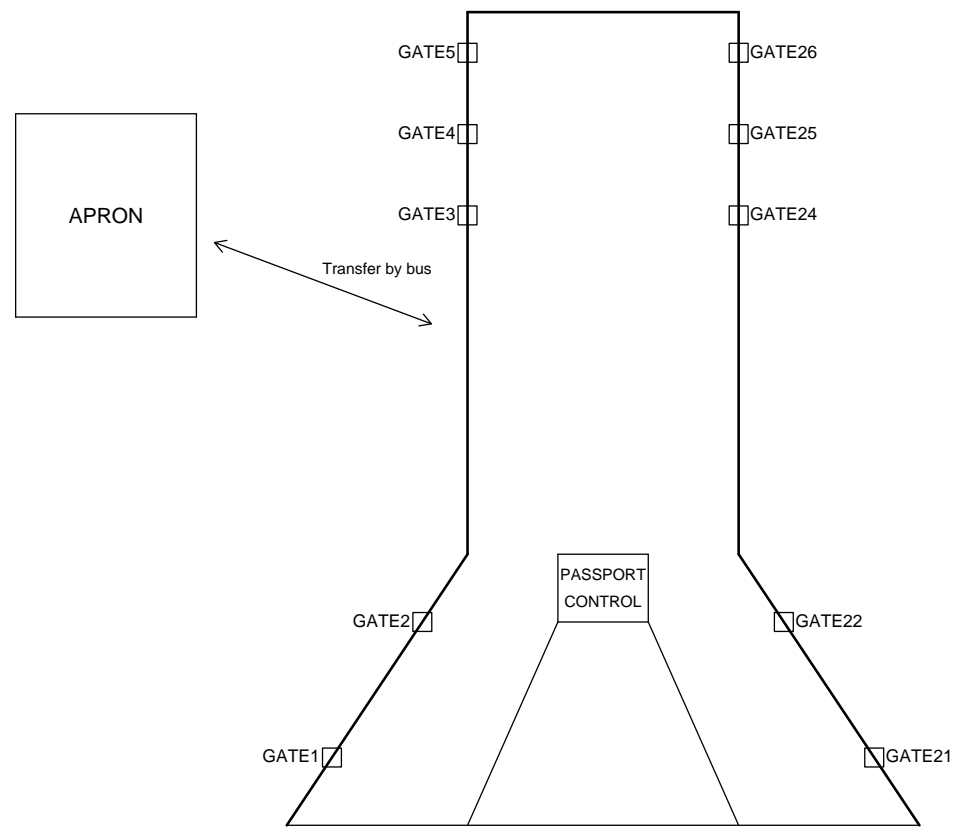

Figure 1: KIA's gates terminal

Table 1: Daily movement

\begin{tabular}{cccccccccc}
\hline \hline \multirow{2}{*}{ A/L } & \multirow{2}{*}{ A/C } & \multirow{2}{*}{ FLT\# } & \multicolumn{2}{c}{ Arrival } & Incoming & \multirow{2}{*}{ FLT \# } & \multicolumn{2}{c}{ Depature } & \multirow{2}{*}{ Outgoing } \\
\cline { 7 - 8 } & & From & time & Gate & To & time & Gate \\
\hline JAI & 737 & 574 & COK & 0040 & 25 & 573 & COK & 0140 & 25 \\
RJA & 319 & 5256 & AMM & 0045 & 3 & 5257 & AMM & 0130 & 3 \\
MEA & 320 & 408 & BEY & 0110 & 21 & 409 & BEY & 0200 & 21 \\
THY & 737 & 1172 & IST & 0115 & 26 & 1173 & IST & 0215 & 26 \\
MSR & 737 & 614 & CAI & 0145 & 22 & 615 & CAI & 0245 & 22 \\
$\vdots$ & $\vdots$ & $\vdots$ & $\vdots$ & $\vdots$ & $\vdots$ & $\vdots$ & $\vdots$ & $\vdots$ & $\vdots$ \\
JZR & 320 & 489 & DAM & 2125 & 4 & 188 & DXB & 2220 & 4 \\
JZR & 320 & 415 & BEY & 2200 & 5 & 636 & ALP & 2320 & 5 \\
JZR & 320 & 407 & DXB/BAH & 2210 & 3 & 502 & LXR & 2315 & 3 \\
JZR & 320 & 185 & DXB & 2240 & 25 & 516 & HRG & 2325 & 25 \\
\hline \hline
\end{tabular}

- $\mathrm{A} / \mathrm{L}$ (airline)

- $\mathrm{A} / \mathrm{C}$ (type of aircraft)

- FLT $\sharp$ (flight number)

- Arrival from / departure to

- GATE (Gate number for arrival / departure flight)

From the collected data:

- We have 756 arrival flights and 754 departure flights. 
Table 2: Types of aircraft that could be assigned to each gate in the terminal

\begin{tabular}{|c|c|c|c|c|c|c|c|c|c|c|}
\hline & Gate1 & Gate2 & Gate3 & Gate4 & Gate5 & Gate21 & Gate22 & Gate24 & Gate25 & Gate26 \\
\hline 310 & $\bigcirc$ & $\bigcirc$ & $\bigcirc$ & $\bigcirc$ & $\bigcirc$ & $\bigcirc$ & $\bigcirc$ & $\bigcirc$ & $\bigcirc$ & $\bigcirc$ \\
\hline 319 & $\bigcirc$ & 0 & 0 & $\bigcirc$ & 0 & 0 & 0 & 0 & 0 & 0 \\
\hline 320 & $\bigcirc$ & $\bigcirc$ & 0 & $\bigcirc$ & 0 & 0 & $\bigcirc$ & $\bigcirc$ & 0 & 0 \\
\hline 321 & $\bigcirc$ & $\bigcirc$ & $\bigcirc$ & $\bigcirc$ & $\bigcirc$ & $\bigcirc$ & $\bigcirc$ & $\bigcirc$ & $\bigcirc$ & 0 \\
\hline 300 & & $\bigcirc$ & $\bigcirc$ & $\bigcirc$ & $\bigcirc$ & $\bigcirc$ & $\bigcirc$ & $\bigcirc$ & $\bigcirc$ & $\bigcirc$ \\
\hline 330 & & $\bigcirc$ & 0 & $\bigcirc$ & 0 & 0 & $\bigcirc$ & & & $\bigcirc$ \\
\hline 340 & & 0 & 0 & 0 & 0 & 0 & 0 & & & 0 \\
\hline 727 & $\bigcirc$ & 0 & 0 & 0 & 0 & 0 & 0 & $\bigcirc$ & $\bigcirc$ & 0 \\
\hline 737 & $\bigcirc$ & 0 & 0 & 0 & 0 & 0 & 0 & 0 & 0 & 0 \\
\hline 747 & & $\bigcirc$ & & $\bigcirc$ & $\bigcirc$ & $\bigcirc$ & $\bigcirc$ & & & $\bigcirc$ \\
\hline 767 & & 0 & 0 & 0 & 0 & 0 & 0 & & 0 & 0 \\
\hline 777 & & $\bigcirc$ & 0 & $\bigcirc$ & 0 & 0 & 0 & & & \\
\hline DC10 & & $\bigcirc$ & $\bigcirc$ & $\bigcirc$ & $\bigcirc$ & $\bigcirc$ & $\bigcirc$ & & & $\bigcirc$ \\
\hline MD90 & & 0 & 0 & 0 & 0 & 0 & 0 & & $\bigcirc$ & 0 \\
\hline
\end{tabular}

- From the arrival flights, we have 212 flights ungated (which means that $28.04 \%$ from the arrival flights are ungated).

- From the departure flights, we have 30 flights ungated(which means that $25.60 \%$ from the departure flights are ungated).

Table 2 presents the types of aircraft that could be assigned to each gate in the terminal. We can notice that Gate 1 is the smallest gate in the terminal since it has the smallest number of types of aircraft which can be assigned to it. On the other hand, Gate 2, Gate 4, Gate 5, Gate 21 and Gate 22 are considered the largest gates in the terminal since they could be use by any type of aircraft.

\section{Problem Description and Model Formulation}

In this project, we consider the airport gate assignment problem(AGAP), where the number of flights exceeds the number of gates available. Our objective is to minimize the number of ungated flights or minimize the number of flights assigned to the apron and the total walking distance or connection times. We will consider the size of each gate in the terminal. We represent $\omega_{i}$ set of gates that can be assigned to flight. According to KIA officials, the towing process for the aircraft will be applied if this aircraft is scheduled to a specific gate for more than 6 hours. The towing process means pulling off an aircraft from the terminal gate to the aircraft stand area. The same process will be used to pull off an aircraft from the stand area to the terminal gate for departure. According to the KIA officials, the towing process takes approximately one hour. One of the reasons for using the towing process is if a flight is scheduled to use a gate for more than 6 hours, we pull off this aircraft from the assigned gate after one hour from its arrival to give an opportunity to other aircraft to use this gate. In addition, we will add a buffer time that is added between two continuous flights assigned to the same gate. The time interval locked for particular aircraft is equal to $\left[a_{i}, d_{i}+\alpha\right]$. According to KIA officials, the buffer time is one hour for each flight.

\subsection{Identify decision variables}

Notations:

$N$ : Represent set of flights arriving to/departing from the airport.

$M$ : Represents set of gates available at the airport. 
$n$ : Total number of flights.

$m$ : Total number of gates.

$a_{i}$ : Arrival time of flight $i$.

$d_{i}$ : Departure time of flight $i$.

$f_{i, j}$ : Number of passengers transferring from flight $i$ to flight $j$.

$w_{k, l}$ : Walking distance for passengers from Gate $k$ to Gate $l$.

$\alpha$ : Buffer time that locks the gate before aircraft's arrival and after its departure (0-1 hour).

$\omega_{i}$ : Represents set of gates that can be assigned to flight $i$.

DF: the difference between the departure and the arrival time (6 hours).

TP: The towing process (1 hour).

Additionally, we will make use of two dummy gates. Gate 0 represents the entrance or exit of the airport, and gate $m+1$ represents the apron where flights arrive when no gates are available. Hence, $w_{k, 0}$ represents the walking distance between Gate $k$ and the airport entrance or exit, and $f_{0, i}$ represents the number of originating departure passengers of flight $i ; f_{i, 0}$ represents number of the disembarking arrival passengers of flight $i$. So $w_{m+1, k}$ represent the walking distance between the apron and Gate $k$ (usually significantly larger than the distance among different gates).

The binary variables

$$
y_{i, k}= \begin{cases}1, & \text { if flight } i \text { is assigned to Gate } k(0<k \leq m+1), \\ 0, & \text { otherwise. }\end{cases}
$$

The following constraint must be satisfied:

$$
\begin{aligned}
& \forall(i, j), \quad k \in \omega_{i} \cap k \in \omega_{j}, \\
& y_{i, k}=y_{j, k}=1(k \neq m+1) \text { Implies } a_{i}>d_{j} \cup a_{j}>d_{i} .
\end{aligned}
$$

This condition disallows any two flights to be scheduled to the same gate simultaneously (except if they are scheduled to the apron).

\subsection{Constraints and objective function}

Our objective is to minimize the number of flights assigned to the apron and the total walking distance. The mathematical formulation can be expressed as follow:

$$
\begin{array}{ll}
\text { Minimize } & \sum_{i=1}^{n} y_{i, m+1}, \\
\text { Minimize } & \sum_{i=1}^{n} \sum_{j=1}^{n} \sum_{k=1}^{m+1} \sum_{l=1}^{m+1} f_{i, j} w_{k, l} y_{i, k} y_{j, l}+\sum_{i=1}^{n} f_{0, i} w_{0, i}+\sum_{i=1}^{n} f_{i, 0} w_{i, 0} .
\end{array}
$$


Equation (3.1) refers to the first objective that minimizes the number of flights assigned to the apron. And Equation (3.2) refers to the second objective which is minimizes the total walking distance. We will call the value of Equation (3.2) the walking distance cost. The constraints:

1. Ensures that every flight must be assigned to one and only one gate or assigned to the apron.

$$
\sum_{k \in \omega_{i}} y_{i, k}=1, \quad(\forall i, 1 \leq i \leq n) .
$$

Where $\omega_{i}$ represent set of gates that can be assigned to flight $i$.

2. Each flight's departure time is later than its arrival time.

$$
a_{i}<d_{i}, \quad(\forall i, 1 \leq i \leq n) .
$$

3. Two flights schedule cannot overlap if they are assigned to the same gate.

$$
y_{i, k} y_{j, k}\left(d_{j}-a_{i}\right)\left(d_{i}-a_{j}\right) \leq 0, \quad\left(\forall i, j, k \in \omega_{i} \cap k \in \omega_{j}, 1 \leq i, j \leq n, k \neq m+1\right), \quad y_{i, k} \in\{0,1\} .
$$

\section{Algorithm and Data Generation}

To solve the AGAP, we will use greedy algorithm which uses a heuristic methods for minimizing the number of flights assigned to the apron. The basic details of the algorithm are as follow:

1. Sort the flights according to the departure time $d_{i}(1 \leq i \leq n)$. Let $g_{k}(1 \leq k \leq m)$ represents the earliest available time (actually the departure time of last flight) of Gate $k$. Set $g_{k}=-1$ for all $k$.

2. For each flight $i$

- Find Gate $k$ such that $g_{k}<a_{i}$ and $g_{k}$ is maximized; and $k \in \omega_{i}$.

- If such $k$ exists, assign flight $i$ to Gate $k$, update $g_{k}=d_{i}$.

- If $k$ does not exist, assign flight $i$ to the apron.

3. Output the result.

Note that in step 2 before assigning flight $i$ to Gate $k$, we will check if $d_{i}-a_{i}>\mathrm{DF}=6$ hours. If the answer is yes, we will divide this flight into two flights and apply the towing process which will take $\mathrm{TP}=1$ hour. The first flight's time interval becomes $\left[a_{i}, a_{i}+1\right]$ and the second flight's time interval become $\left[d_{i}-1, d_{i}\right]$. This means if a flight is scheduled to use a gate for more than 6 hours, we pull off this aircraft from the assigned gate after one hour from its arrival to give an opportunity to other aircraft to use this gate. Then for this flight's departure, if we find a gate, we will assign this flight to this gate one hour before its departure.

Proof of the correctness of the greedy algorithm: By induction, assume we have found the optimum solution after scheduling flight $i$ by the greedy algorithm. Now by this, we will assign flight $f$ to Gate $k$. But the optimal solution is to drop flight $f$ and assign $f^{\prime}\left(f^{\prime}>f\right)$ to Gate $k^{\prime}$. Hence we can always replace $f^{\prime}$ by $f$ to make our greedy solution no worse than the optimal solution. There are two cases we should consider:

1. If $k=k^{\prime}$, since we sort the flight by departure time, $d_{f} \leq d_{f^{\prime}}$. We have $g_{k} \leq g_{k}^{\prime}$. As we considered the earliest available time of the gates, we find the greedy solution is better or at least equal to the optimal solution. 


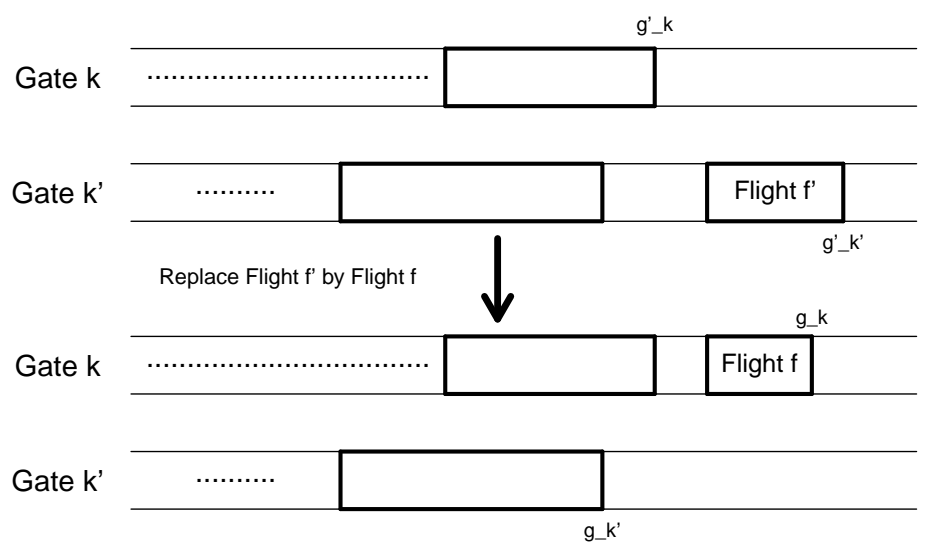

Figure 2: The correctness of the greedy algorithm

Table 3: Walking distance

\begin{tabular}{cc}
\hline \hline GATE & DISTANCE (in units) \\
\hline 1,21 & 2 \\
2,22 & 1 \\
3,24 & 4 \\
4,25 & 5 \\
5,26 & 6 \\
APRON & 8 \\
\hline \hline
\end{tabular}

2. If $k \neq k^{\prime}$, we find that $g_{k} \leq g_{k^{\prime}}^{\prime}$ and $g_{k^{\prime}} \leq g_{k}^{\prime}$, since we choose the maximum $g_{k}$ in the greedy solution. The Figure 2 illustrates this.

The arrival time and the departure time for flight $i$ are actual data from the schedule department at Kuwait international airport. But other data should be generated or assumed to apply our model. First for the walking distance, we assume that the distance measure between two gates which are next to each other is 1 unit. For example, if one passenger arrived at Gate 25 his walking distance to the passport control is 5 units (The distance measure is known as Manhattan Distance). Table 3 represents a summary for the assumed walking distance from a specific gate to the passport control.

We can use Table 3 to assume the walking distance from Gate $k$ to the airport entrance or exit $\left(w_{k, 0}\right)$ or the walking distance from the airport entrance or exit to Gate $k\left(w_{0, k}\right)$. For the transferring passengers, the walking distance from Gate $k$ to Gate $l\left(w_{k, l}\right)$ is randomly generated in the interval $[1,8]$. Now for the incoming passengers $f_{i, 0}$ and the departure passengers $f_{0, i}$ are randomly generated from different interval sizes depending on the type of the aircraft. Table 4 represents the scenarios to generate the incoming and the departure passengers.

There are rarely small numbers of passengers transferring from one flight to another flight. The number of transfer passengers will increase if flight schedules are close, but not too close (At least 1 hour different). The number of transferring passengers from flight $i$ to flight $j\left(f_{i, j}\right)$ is usually within a certain interval, say $[1,50]$.

\section{Results}

We implement R (statistical software) to solve the problem. The detailed of the results and analysis are presented in the next section. 
Table 4: Incoming and the departure passenger's generation

\begin{tabular}{cc}
\hline \hline Type & Data Generation \\
\hline 310 & {$[180,280]$} \\
319 & {$[80,126]$} \\
320 & {$[80,180]$} \\
321 & {$[86,186]$} \\
300 & {$[235,335]$} \\
330 & {$[235,335]$} \\
340 & {$[195,295]$} \\
727 & {$[87,187]$} \\
737 & {$[89,189]$} \\
747 & {$[324,424]$} \\
767 & {$[88,188]$} \\
777 & {$[244,344]$} \\
DC10 & {$[270,370]$} \\
MD90 & {$[87,187]$} \\
\hline
\end{tabular}

Table 5: The output of the result

\begin{tabular}{|c|c|c|c|c|c|c|}
\hline \multirow{2}{*}{$\mathrm{A} / \mathrm{L}$} & \multirow{2}{*}{$\mathrm{A} / \mathrm{C}$} & \multicolumn{2}{|c|}{ Arrival } & \multirow{2}{*}{ Gate } & \multicolumn{2}{|c|}{ Departure } \\
\hline & & From & time & & To & time \\
\hline $\mathrm{AXB}$ & 737 & - & - & 2 & TRV/CCJ & 10 \\
\hline SYZ & 320 & - & - & 1 & DAM & 20 \\
\hline MSR & 320 & - & - & 4 & LXR & 30 \\
\hline LZB & 320 & - & - & 5 & $\mathrm{BOJ}$ & 40 \\
\hline IAC & 320 & - & - & 21 & BOM/MAA & 50 \\
\hline$\vdots$ & : & : & $\vdots$ & $\vdots$ & $\vdots$ & $\vdots$ \\
\hline RJA & 310 & RJA & 2310 & 1 & - & - \\
\hline KLM & 330 & KLM & 2315 & Apron & - & - \\
\hline JZR & 320 & JZR & 2350 & 3 & - & - \\
\hline PIA & 310 & PIA & 2355 & 22 & - & - \\
\hline
\end{tabular}

\subsection{Result of objective 1}

We will apply the greedy algorithm to obtain initial feasible solutions for the first objective function (minimize the number of flights assigned to the apron). Table 5 represents a sample for the output of the result. From the output, after applying the greedy algorithm, the total number of ungated flights for $\alpha=0$ is 85 for the incoming flights and 82 for the outgoing flights. The difference between the actual data and the output is 127 for the incoming flights and 111 for the outgoing flights. Note that the number of the ungated flights for the actual data is 212 in the incoming flights and 193 in the outgoing flights. Table 6 shows the output results for using the greedy algorithm and we have compared these results with the actual data to get the saving percentage. We tried $\alpha=0,10,20,30,40$ and 60 minutes. The results give positive results until we let $\alpha=40$ minutes. The saving percentage is positive for the incoming flights but it becomes negative for outgoing flights.

\subsection{Result of objective 2}

After applying the greedy algorithm, we will use the previous output as shown in table to find the solution for the second objective function (minimize the total walking distance). To do this, we must generate random data for the number of passengers and walking distance as explained in the previous section (Data generation). Table 7 represents the sample for the generated data for the number of passengers and the walking distance.

Before applying the greedy algorithm to get an initial feasible solution for the first objective func- 
Table 6: Output analysis

\begin{tabular}{|c|c|c|c|c|c|c|}
\hline & \multicolumn{2}{|c|}{$\alpha=0$} & \multicolumn{2}{|c|}{$\alpha=10$} & \multicolumn{2}{|c|}{$\alpha=20$} \\
\hline & Incoming & Outgoing & Incoming & Outgoing & Incoming & Outgoing \\
\hline Greedy algorithm & 85 & 82 & 112 & 113 & 135 & 136 \\
\hline Difference from the actual data & 127 & 111 & 100 & 80 & 77 & 57 \\
\hline \multirow[t]{3}{*}{ Saving percentage } & $59.91 \%$ & $57.51 \%$ & $47.17 \%$ & $41.45 \%$ & $36.32 \%$ & $29.53 \%$ \\
\hline & \multicolumn{2}{|c|}{$\alpha=30$} & \multicolumn{2}{|c|}{$\alpha=40$} & \multicolumn{2}{|c|}{$\alpha=60$} \\
\hline & Incoming & Outgoing & Incoming & Outgoing & Incoming & Outgoing \\
\hline Greedy algorithm & 168 & 168 & 196 & 196 & 243 & 244 \\
\hline Difference from the actual data & 44 & 25 & 16 & -3 & -31 & -51 \\
\hline Saving percentage & $20.75 \%$ & $12.95 \%$ & $7.55 \%$ & $-1.55 \%$ & $-14.62 \%$ & $-26.42 \%$ \\
\hline
\end{tabular}

Table 7: Generated data for the number of passengers and the walking distance.

\begin{tabular}{ccccccccccc}
\hline \hline \multirow{2}{*}{$\mathrm{A} / \mathrm{L}$} & \multirow{2}{*}{$\mathrm{A} / \mathrm{C}$} & \multicolumn{2}{c}{ Arrival } & \multirow{2}{*}{$f_{0, j}$} & Gate & \multirow{2}{*}{$w_{i, j}$} & $f_{i, j}$ & $f_{i, 0}$ & \multicolumn{2}{c}{ Departure } \\
\cline { 8 - 10 } AXB & 737 & - & - & 0 & 2 & 2 & 0 & 178 & TRV/CCJ & time \\
SYZ & 320 & - & - & 0 & 1 & 1 & 0 & 137 & DAM & 20 \\
MSR & 320 & - & - & 0 & 4 & 4 & 0 & 127 & LXR & 30 \\
LZB & 320 & - & - & 0 & 5 & 5 & 0 & 99 & BOJ & 40 \\
IAC & 320 & - & - & 0 & 21 & 6 & 0 & 140 & BOM/MAA & 50 \\
DLH & 330 & - & - & 0 & 2 & 2 & 0 & 322 & FRA & 55 \\
AFG & 310 & - & - & 0 & 1 & 1 & 0 & 236 & KBL & 100 \\
KAC & 320 & BEY & 5 & 80 & 4 & 4 & 0 & 0 & - & 105 \\
RJA & 319 & AMM & 45 & 107 & 5 & 5 & 44 & 97 & AMM & 130 \\
$\vdots$ & $\vdots$ & $\vdots$ & $\vdots$ & $\vdots$ & $\vdots$ & $\vdots$ & $\vdots$ & $\vdots$ & $\vdots$ & $\vdots$ \\
RJA & 310 & RJA & 2310 & 212 & 1 & 2 & 0 & 0 & - & - \\
KLM & 330 & KLM & 2315 & 327 & Apron & 8 & 0 & 0 & - & - \\
JZR & 320 & JZR & 2350 & 154 & 3 & 4 & 0 & 0 & - & - \\
PIA & 310 & PIA & 2355 & 246 & 22 & 1 & 0 & 0 & - & - \\
\hline \hline
\end{tabular}

Table 8: The walking distance cost change comparison with the buffer time $\alpha=0$

\begin{tabular}{cccccc}
\hline \hline & $\alpha=10$ & $\alpha=20$ & $\alpha=30$ & $\alpha=40$ & $\alpha=60$ \\
\hline The estimated walking distance cost & 1362301 & 1426177 & 1497324 & 1576803 & 1710229 \\
percentage change & $7.42 \%$ & $12.46 \%$ & $18.07 \%$ & $24.34 \%$ & $34.86 \%$ \\
\hline \hline
\end{tabular}

tion, we have estimated the walking distance cost for the actual data by generating random data for the number of passengers and walking distance. The estimated cost was 1,509,752. After applying the greedy algorithm and generating random data for the number of passengers and walking distance with a buffer time $\alpha=0$, the walking distance costs was $1,268,182$. This means that the walking distance saving percentage is $16.00 \%$. Table 8 represents the walking distance cost change comparison with the buffer time $\alpha=0$.

\section{Conclusion}

In this paper, we considered the over constrained the AGAP(airport gate assignment problem) to minimize the number of flights assigned to the apron while minimizing the total walking distances. We provide a greedy algorithm that minimizes the number of flights not assigned to gates. This algorithm can allocate the flights that will be ungated as well as provide an initial feasible solution while putting in our considerations for the size of each gate in the terminal, the towing process for the aircraft and the aircraft capacity. In addition, we added some analysis for the buffer time which is the 
time that locks the aircraft gate after departure for to minimize the number of flights assigned to the apron and minimizing the total walking distances by estimating the walking distance cost. In future work, further research will be conducted in order to extend the report work. Such as gate utilization (usage percentage) and aircraft's delays forecasting for both arrival and departure flights.

\section{References}

Al-Sultan, A. T., Ishioka, F. and Kurihara, K. (2010). Optimizing gate assignments at airport terminal, JKSC 2010 Joint Meeting of Japan - Korea Special Conference of Statistics and the 2nd Japan Korea Statistics Conference of Young Researchers, 159-166.

Babica, O., Teodorovic, D. and Tosic, V. (1984). Aircraft stand assignment to minimize walking, Journal of Transportation Engineering, 110, 55-66.

Braaksma, J. and Shortreeda, J. (1971). Improving airport gate usage with critical path method, Transportation Engineering Journal of ASCE 97, 187-203.

Cheng, Y. (1998a). Arule-based reactive model for the simulation of aircraft on airport gates, Knowle dge-Based Systems, 10, 225-236.

Cheng, Y. (1998b). Network-based simulation of aircraft at gates in airport terminals, Journal of Transportation Engineering, 188-196.

Ding, H., Lim, A., Rodrigues, B. and Zhu, Y. (2004). Aircraft and gate scheduling optimization at airports, 37th Hawaii International Conference on System Sciences, 3, 30074b.

Haghnani, A. and Chen, M. C. (1998). Optimizing gate assignments at airport terminals, Transportation Research Part A: Policy and Practice, 32, 437-454.

Obata, T. (1979). The quadratic assignment problem: Evaluation of exact and heuristic algorithms, Tech. Report TRS- 7901, Rensselaer Polytechnic Institute, Troy, New York.

$\mathrm{Xu}$, J. and Bailey, G. (2001). The airport gate assignment problem: Mathematical model and a Tabu search algorithm, 34th Hawaii International Conference on System Sciences, 3, 3032.

Yan, S. and Chang, C. M. (1998). A network model for gate assignment, Journal of advanced Transportation, 32, 176-189.

Yan, S. and Huo, C. M. (2001). Optimization of multiple objective gate assignments, Transportation Research Part A: Policy and Practice, 35, 413-432.

Yan, S., Tang, C. H. and Fu, T. C. (2008). An airline scheduling model and solution algorithms under stochastic demands, European Journal of Operational Research, 190, 22-39. 